\title{
Robot Control System Based on Electrooculography and Electromyogram
}

\author{
Minoru Sasaki', Muhammad Syaiful Amri Bin Suhaimi1, Kojiro Matsushita1, Satoshi Ito', \\ Muhammad Ilhamdi Rusydi ${ }^{2}$ \\ ${ }^{1}$ Department of Mechanical Engineering, Gifu University, Gifu, Japan \\ ${ }^{2}$ Department of Electrical Engineering, Andalas University, Padang, Indonesia \\ Email: sasaki@gifu-u.ac.jp,u3128026@edu.gifu-u.ac.jp,kojirom@gifu-u.ac.jp, \\ satoshi@gifu-u.ac.jp,rilhamdi@yahoo.com
}

Received September 2015

\begin{abstract}
This paper describes a man-machine interface system using EOG and EMG. A manipulator control system using EOG and EMG is developed according to EOG and EMG. With the eye movement, the system enabled us to control a manipulator. EOG is using for moving the robot joint angles and EMG is using for object grasping. The EOG and EMG discrimination method is used to control the robot. The robot arm joint movements are determined by the EOG discrimination method where the polarity of eye gaze motion signals in each $\mathrm{Ch} 1$ and $\mathrm{Ch} 2$. The EMG discrimination method is used to control arm gripper to grasp and release the target object. In the robot control experiment, we are successfully control the uArm ${ }^{\mathrm{TM}}$ robot by using both EOG and EMG discrimination method as the control input. This control system brings the feasibility of man-machine interface for elderly person and handicapped person.
\end{abstract}

\section{Keywords}

EOG, EMG, Man-Machine Interface, Robot Control

\section{Introduction}

Recently, machine and robot technology has progress rapidly, improving human live and making human work become easier. Unfortunately most of the conventional machine and robot are usually controlled by hand manipulation and have complicated control system. By the year 2014, the world's population has reach above 7 billion human but in that 7 billion human there are quite a percentage of people with disabilities and aged people. For example, in Japan alone, based on year 2009 report, the people who are with disabilities and need supports are about 4,690,000. For those aged people and people with disabilities, the ever growing machine and robot technology seem unfeasible to make use of it. The people with disabilities especially those who have physical disabilities, the hand manipulation based control system and the complicated control system are unusable for them. As for aged people, the human aging is closely related to the decrease of the mass and the voluntary contractile of muscle and this condition often causes a disability condition among older people. To overcome this problem with the machine and robot control system, human machine interface technology has being research 
progressively to solve the problem. In the human machine interface there many ways of control system exists and one of the prominent ways is by using the bio-signal.

The bio-signals are a type of signal that could be obtained by any living being. In the bio-signal, the bio-signal that are usually used as medium of communication for human and machine is electrooculography (EOG) and Electromyography (EMG). Electrooculography (EOG) is a method for measuring the cornea-retinal electrical potential that exists between the front and the back of the eye. Electromyography (EMG) in other hand is a method for obtaining the electrical potential produced by human muscles.

Performing some special tasks using EOG and EMG in daily activities is being developed in various areas. These inventions improve the life quality of people in human machine interface. EOG is recorded by attaching electrodes to the skin around the subject's eyes. As for EMG, the signal is recorded by attaching electrodes on effected muscle area that have relationship with human mastication or biting motion. Both of the EOG and EMG research used NF Instrument as the signal recording device. This device has an amplifier (EEG head box), a processors box, and four electrodes. The electrodes consist of one ground electrodes, one reference electrodes, channel 1 (Ch1) and channel 2 (Ch2) for recording both EOG and EMG signal simultaneously. Three digital filters were given by the processor box, which are $1.6 \mathrm{~Hz}$ High Pass Filter, $30 \mathrm{~Hz}$ Low-Pass Filter and $60 \mathrm{~Hz} \mathrm{Ham}$ Filter. The $30 \mathrm{~Hz}$ Low-Pass Filter was added to reduce the electricity equipment noise beside effectively record the EMG signal produced from biting motion. The EMG signal theoretically has higher amplitude than EOG signal, the NF Instrument EEG head box used for the research could not record the EMG signal effectively unless the low-pass filter is set to lower value. There are three basic steps in human machine interface using both bio-signals, amplifying and acquisition of signal from human, pattern recognition of the signals and action to control a machine using the signal. In previous studies [1]-[11], we have worked on electrooculography (EOG) and applied EOG-based-control for a robot arm. However, EOG is suitable for directional control, but unsuitable for grasping objects. Therefore, we propose robot-arm control based on both EOG and EMG signals: EOG is for moving the robot joint angle and EMG is for object grasping.

\section{Type of Bio-Signal}

\subsection{Electrooculography (EOG)}

The electrooculography (EOG) is a type of bio-signal that have relationship with eye movement. Electrooculography (EOG) is a method for measuring the cornea-retinal electrical potential that exists between the front and the back of the eye. The frontal part of the eye cornea produced positive electrical potential and the back part of the eye retina produced the negative electrical potential. One of the merit of the signal is the signal is easily to be recorded if the electrode is positioned around one's eye. The demerit of the signal produced a lot of noises, the signal is need to be filtered to get good signal for the EOG. Besides that, if a subject doing EOG for a certain long period, the signal produced will also have many noises.

\subsection{Electromyogram (EMG)}

The electromyogram (EMG) is a bio-signal that associated with the electrical potential from human muscle. There are many ways to get the EMG, from moving one's hand to moving one's head, depending on a motion that associated with the use of one's muscle. In my research, I have used mastication or biting motion as a way to produce EMG signal. To determine the EMG for the study, we need to understand which muscle used for biting motion. The muscle used primarily is around one's mouth. Usually, the muscle used Masseter, Buccinator, Zygomaticus minor/major and depressor anguli oris as shown in Figure 1. Human mastication or biting motion also depend on one age, race, area of reside and eating habit.

\section{Research Equipment}

\subsection{Bio-Signals Instrument}

NF Instrument is an equipment to detect and record signals. It can be used to get both EOG and EMG signal. NF instrument as shown in Figure 2 have 2 boxes, one is processor box and other is EEG head box. Both of the boxes have different function. Head box is used to amplify signal to send the data from operators to processor box. Processor box is used to provide some specification of filters that could be used for the research. 


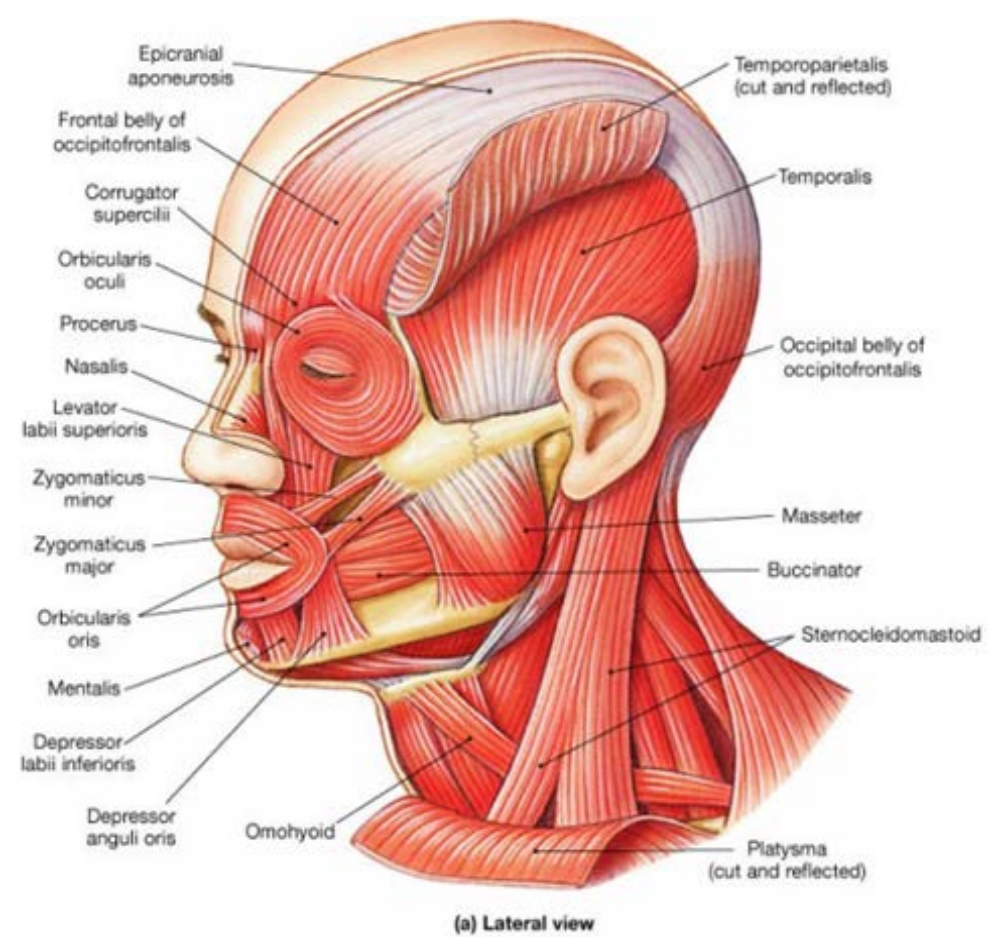

Figure 1. Human face muscles (Ref.: http://www.advocurenf2.org/livingwithnf2_ailments+care_face.php).
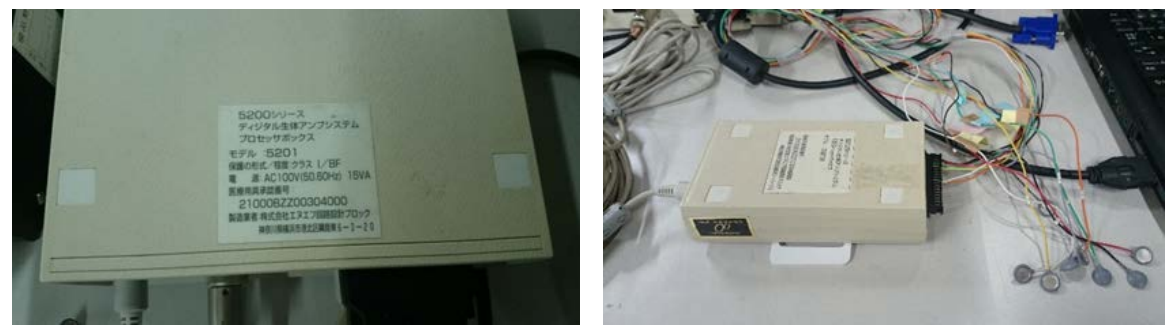

Figure 2. NF instrument processor box (Left picture) and EEG head box with electrodes (Right picture).

\section{2. uArm $^{\mathrm{TM}}$}

The robot used for the research is uArm ${ }^{\mathrm{TM}}$ robot as shown in Figure 3. The robot is made from u Factory company from China. The robot used 5 servo motor each motor use to control the motion function of the robot. The motion function consists of moving up and down the robot arm joint, stretch forward and backward robot arm joint, robot base rotation, robot gripper part rotation and gripping and releasing the robot gripper.

\subsection{Visual Studio 2008 and Bio-Signals}

Visual studio 2008 and 2010 express is a software that be used to program a 2010 Express instrument and device based on $\mathrm{C} / \mathrm{C}^{++}$programming language. In this study, the Visual Studio is used as a medium for programming the NF instrument for EOG and signal detection and recording. In addition the visual studio is used to program the $\mathrm{uArm}^{\mathrm{TM}}$ for EOG and EMG robot control system in $\mathrm{C} / \mathrm{C}++$ programming via serial communication.

\section{Research Method}

\subsection{EOG Electrode Position}

In previous study, we have determined the position for detecting and recording the EOG signal. As the EOG has relationship with eye activities, the electrode is positioned around the as shown in Figure 4. In this study, we are searching for the EOG signal characteristic and patterns when doing up, down, left and right gaze motion. 


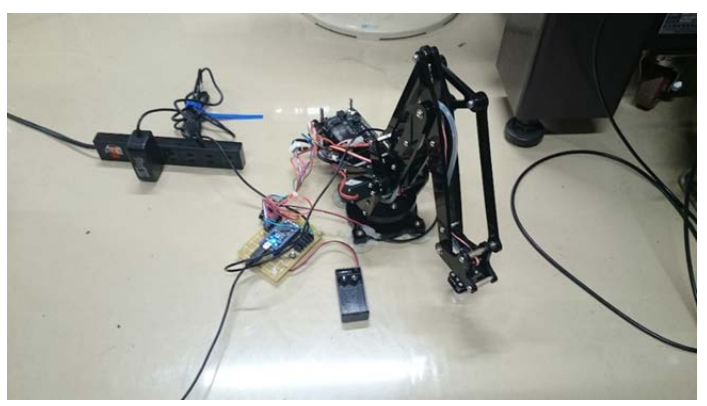

Figure 3. uArm ${ }^{\mathrm{TM}}$ robot.

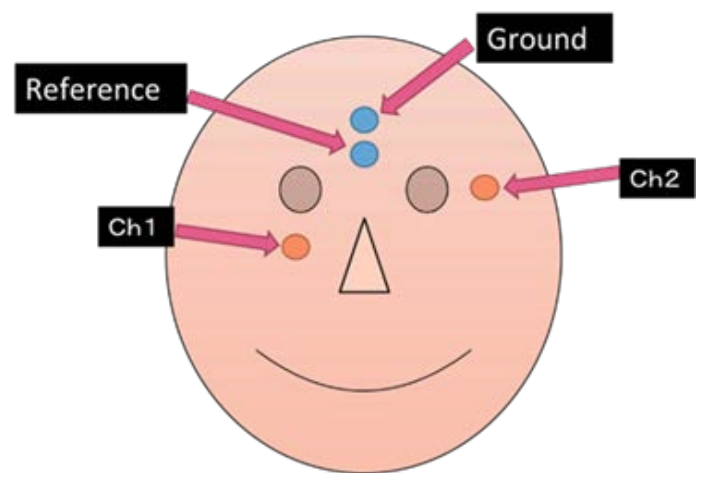

Figure 4. EOG electrodes position.

\subsection{Experimental EMG Electrode Position}

EMG signal could be derived by many way regarding human activities that using any muscle in one's body. In this study we are using mastication or bite motion as a method to obtain the EMG signals as shown in Figures 5-8. In order to obtain those signal, the electrodes are positioned consequently with the face muscle that be used during the biting motion. The target face muscle for this study is Masseter, Buccinator, Depressor anguli oris, and Zygomaticus major/minor. The electrode will position on face skin proportional to the muscle mentioned. The electrode positions are changed for each section and the number of biting motion for each section is three times. The respective result of each muscle electrode position will be derived to find signal characteristic and pattern.

\subsection{EOG and EMG Electrode Position}

As we have determined the location of best location on EOG and EMG respectively, the next step of our study is to determine whether the both EOG and EMG electrode positions could be combined together and get the same signals result as done independently. Basically, 4 electrodes are used in the experiment, the electrode of Ch1 and Ch2 are specialize to detect and record EOG and electrode Ch3 and Ch4 are specialize for EMG. Each electrode position is given a code name perpendicular to their number of electrodes used to discriminate them. The method of obtaining the EOG and EMG signals are the same, eye gazes motion and biting motion. Besides, the sampling rate is $100 \mathrm{~Hz}$ and the low-pass filter used for the experiment is $60 \mathrm{~Hz}$. Each results are used to determine the signals characteristic and pattern.

\subsection{Robot Control System}

The control system for the uArm robot arm is shown in Figure 9. The NF Instrument of processor box and EEG head box are used in detecting and recording the EOG and EMG signal from eye gaze motion and biting motion of a subject. The computer is used to discriminate the EOG and EMG signals respectively and use it as a control input for the robot arm. In addition, the computer also is used for programming the robot arm to do motion functions such as joint arm movement and gripping for each EOG and EMG signals control input. 


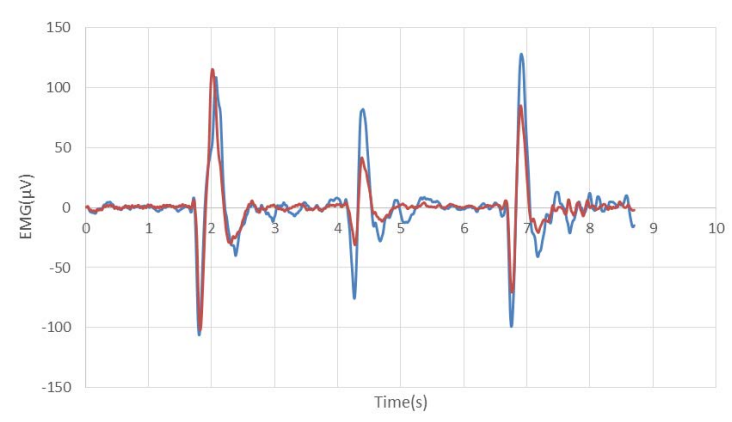

Figure 5. Masseter EMG signal.

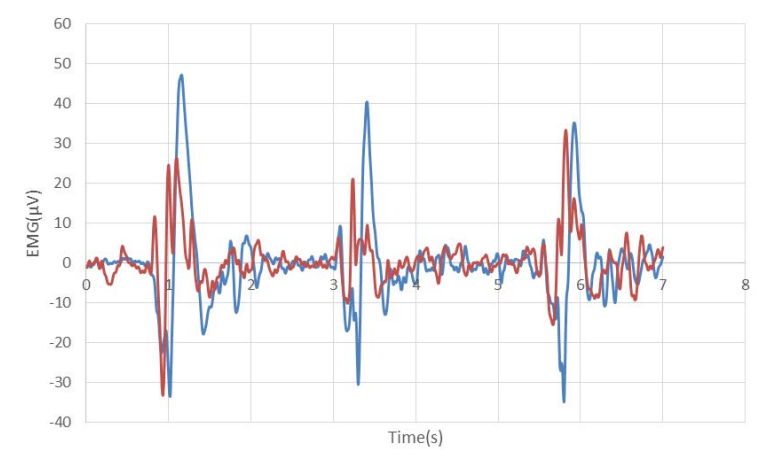

—Ch1 -

Figure 6. Buccinator EMG signal.

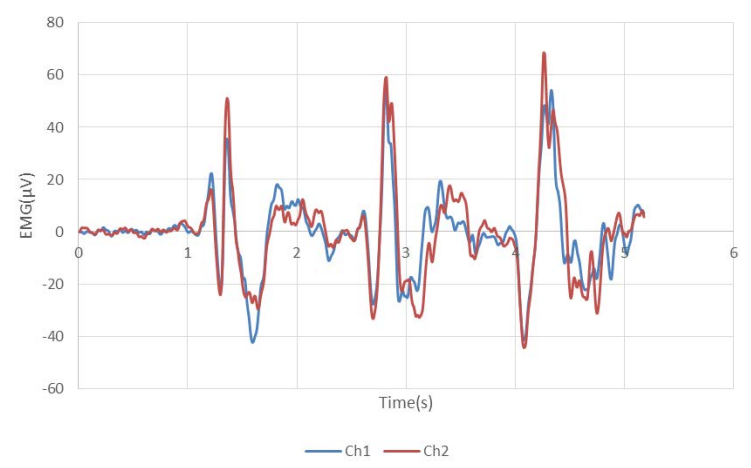

Figure 7. Depressor anguli oris EMG signal.

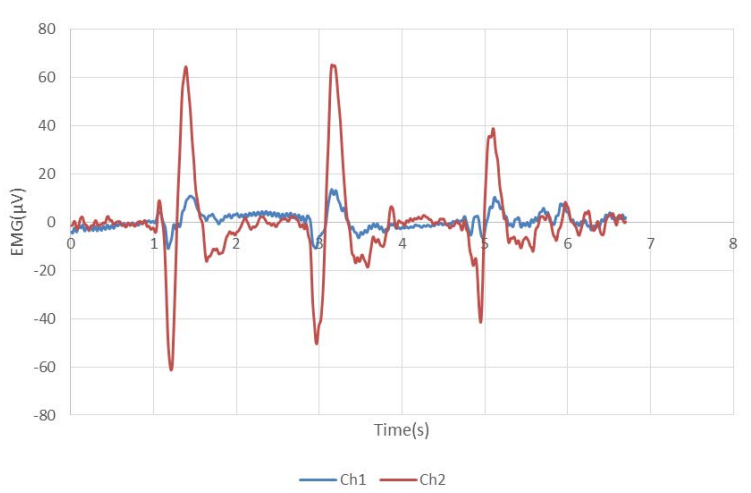

Figure 8. Zygomaticus major/minor EMG signal. 


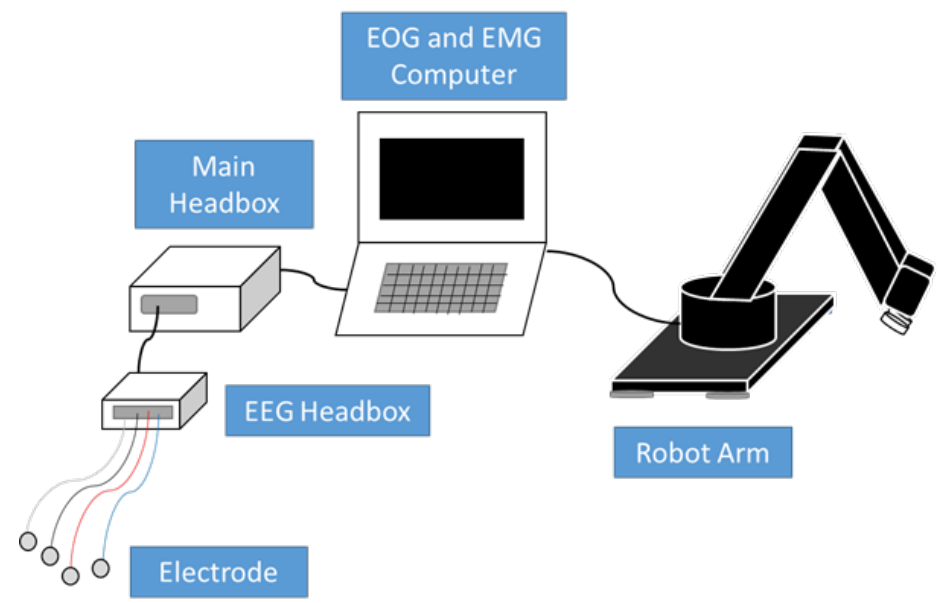

Figure 9. Robot control system.

\section{Result and Discussion}

\subsection{EOG and EMG Electrode Position}

In the combination of EOG and EMG electrode position, the signal recorded especially in Ch3 and Ch4 is unstable and hard to be analyzed. In all experiment, the biting motion signal obtained similar to noise signal. In addition, during doing eye gaze motion, all specialized electrode which used for detecting and recording EOG and EMG reacted to the motion. It is the as for biting motion, all specialized electrode reacted to the motion. Thus, the EOG and EMG signals are hard to discriminate for the robot control system.

From signal result obtained for all the electrode position, we realized that we do not many electrode to detect and record EOG and EMG signal. It is possible if only using EOG electrode position to detect and record both EOG and EMG signal as shown in Figures 10-12. The cut-off frequency for the low-pass filter should be lowered to $30 \mathrm{~Hz}$ in order to record EMG signal effectively.

\subsection{EOG Discrimination Method}

Based on the signals obtained, we could discriminate each gaze motions. The discrimination method for each gaze motions could be determined by the polarity of the first peak amplitude in Ch1 and Ch2 of the electrodes as shown in Tables 1 and 2.

\subsection{EMG Discrimination Method}

As for EMG signal we decided to use strong biting motion as medium to get EMG signal. Strong biting motion has higher magnitude of amplitude than other and has consistent signal result than other. The higher magnitude of amplitude and consistency of EMG signal are essential to discriminate the EMG than EOG signal.

The discrimination method for EMG signal, we done several strong biting motion experiment to investigate the signal characteristic and pattern. By the experiment done, the EMG signal can be discriminate using the Ch2 EMG signal as the Ch2 gave stable and consistent signal.

To discriminate the EMG signal as control input for robot control, we found that the sum and ratio of the signal can be used for this. The sum and ratio of Ch2 EMG signal is much higher in compare with the Ch2 EOG. Table 3 shows the polar peak amplitude of Ch2 EMG signal relationship with sum and ratio of the signal obtained. The equation for the sum and ratio of signal is derived as below.

$$
\begin{aligned}
& \text { Sum }=\mid \text { 『Ch2】_max }|+| \text { 『Ch2】_min } \mid \\
& \text { ratio }=\mid \text { 『Ch2】_max } / \llbracket C h 2 \rrbracket \_m i n \mid
\end{aligned}
$$

where

【Ch2】_max $=$ Positive Peak Amplitude
『Ch2】_min = Negative Peak Amplitude 


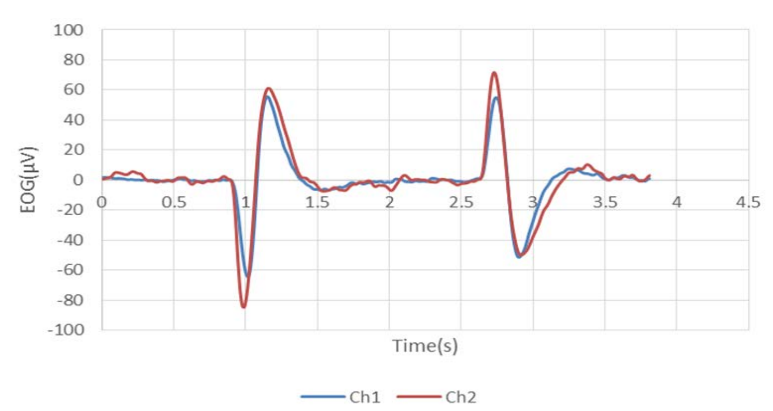

Figure 10. Up and down gaze motion signal.

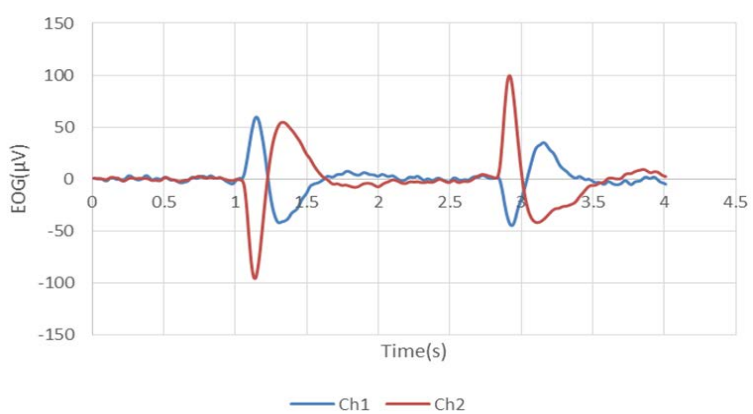

Figure 11. Right and left gaze motion signal.

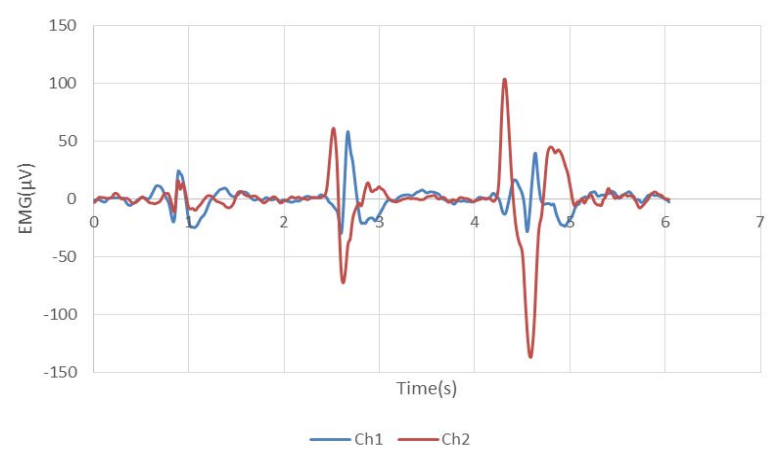

Figure 12. Biting motion strength signal. Left to right; small, normal and strong biting motion signal.

\subsection{Robot Control}

In this part, the EOG and EMG discrimination method is used to control the robot. The robot arm joint movements are determined by the EOG discrimination method where the polarity of eye gaze motion signals in each Ch1 and Ch2. The EMG discrimination method is used to control arm gripper to grasp and release the target object. In the robot control experiment, we are successfully control the uArm robot by using both EOG and EMG discrimination method as the control input.

\section{Conclusions}

In this study we conclude that EOG and EMG signal can be detected and recorded using the EOG electrode position as shown in Figure 4. We successfully archived to measure EMG signals with EOG electrodes by setting the low-pass filter (cut off freq.: 30[Hz]). Besides we were able to classify the EOG and EMG into 5 signal patterns as shown in Figure 13, and use those for robot control input. We also found that the Ch2 sum for EMG signal from the strong biting motion is between $220-180(\mu \mathrm{V})$ and with a signal ratio of between 0.8 and 1.1. The patterns are as shown in Figure 13. 

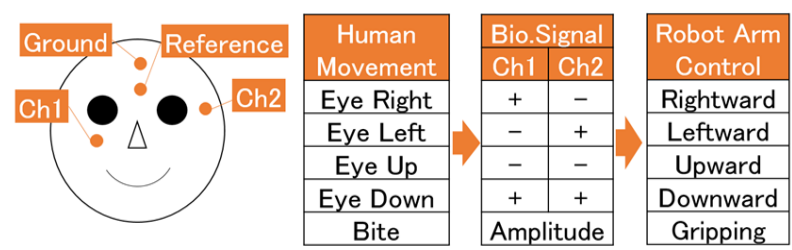

Figure 13. EOG and EMG 5 signal patterns discrimination.

Table 1. EOG gaze motions discrimination result.

\begin{tabular}{ccccc}
\hline & Up & Down & Right & Left \\
Ch1 & + & - & - & + \\
Ch2 & + & - & + & - \\
\hline
\end{tabular}

Table 2. EOG peak amplitudes from 4 EOG gaze motions.

\begin{tabular}{|c|c|c|c|c|c|c|c|c|c|c|c|c|}
\hline Gaze motion & \multicolumn{3}{|c|}{ Up } & \multicolumn{3}{c|}{ Down } & \multicolumn{3}{c|}{ Left } & \multicolumn{3}{c|}{ Right } \\
\hline |Ch2_max & 55.28 & 60.60 & 63.90 & 71.97 & 76.69 & 66.17 & 99.67 & 98.92 & 103.27 & 54.70 & 41.91 & 41.97 \\
\hline |Ch2_min| & 82.66 & 88.17 & 99.49 & 53.67 & 51.56 & 46.16 & 41.61 & 40.60 & 41.15 & 95.53 & 105.68 & 108.15 \\
\hline Sum & 137.94 & 148.77 & 163.39 & 125.64 & 128.25 & 112.33 & 141.28 & 139.52 & 144.42 & 150.23 & 147.59 & 150.12 \\
\hline Ratio & 0.67 & 0.69 & 0.64 & 1.30 & 1.49 & 1.43 & 2.39 & 2.44 & 2.51 & 0.57 & 0.40 & 0.39 \\
\hline
\end{tabular}

Table 3. EMG peak amplitudes from strong bite EMG.

\begin{tabular}{|c|c|c|c|c|c|c|}
\hline No. of Experiment & 1st & 2nd & 3rd & 4rd & 5th & 6th \\
\hline $\mid$ Ch2_Max $\mid$ & 94.84 & 101.13 & 102.02 & 76.71 & 97.76 & 94.22 \\
\hline $\mid$ Ch2_Min $\mid$ & 99.76 & 109.87 & 117.20 & 115.19 & 112.37 & 102.59 \\
\hline Sum & 194.6 & 211.00 & 219.22 & 191.90 & 210.13 & 196.81 \\
\hline Ratio & 0.95 & 0.92 & 0.87 & 0.67 & 0.87 & 0.92 \\
\hline
\end{tabular}

Together with the signal patterns, we now can create a control system using EOG and EMG signal respectively. The EOG signals are used as robot joint angle movement control and EMG is used for controlling the degree of gripping.

\section{References}

[1] Cristian-Cezar, P., Florin, G. and Doru, T. (2012) EOG-Based Visual Navigation Interface Development. Expert Systems with Application, 9, 10857-10866.

[2] Deng, Y.L., Hsu, C.L., Lin, T.C., Tuan, J.S. and Chang, S.H. (2010) EOG-Based Human-Computer Interface System Development. Expert Systems with Application, 37, 3337-3343. http://dx.doi.org/10.1016/j.eswa.2009.10.017

[3] Lledo, L.D., Ubeda, A., Ianez, E. and Azorin, J.M. (2013) Internet Browsing Application Based on Electrooculography for Disabled People. Expert Systems with Applications, 40, 2640-2648. http://dx.doi.org/10.1016/j.eswa.2012.11.012

[4] Bera, R., Boquete, L., Mazo, M. and Lopez, E. (2002) Wheelchair Guidance Strategies Using EOG. Journal of Intelligent and Robotic Systems, 34, 279-299. http://dx.doi.org/10.1023/A:1016359503796

[5] Eduardo, I., Jose, M.A., Eduardo, F. and Andre, U. (2010) Interface Based on Electrooculography for Velocity Control of a Robot Arm. Applied Bionics and Biomechanics, 7, 199-207. http://dx.doi.org/10.1155/2010/813184

[6] Sasaki, M., Ito, S., Takeda, K., Okamoto, T. and Rusydi, M.I. (2013) Developing a Two-Link Robot Arm Controller Using Voluntary Blink. Proceedings of the 22nd MAGDA Conference, Miyazaki, December 2013.

[7] Rusydi, M.I., Okamoto, T., Ito, S. and Sasaki, M. (2014) Rotation Matrix to Operate a Robot Manipulator for 2D Analog Tracking Object Using Electrooculography. Robotics, 3, 289-309. http://dx.doi.org/10.3390/robotics3030289

[8] Rusydi, M.I., Mori, Y., Okamoto, T., Sasaki, M. and Ito, S. (2012) Using EOG Signal to Control Manipulator. Proceedings of the 7th Asia Pacific Symposium on Applied Electromagnetics and Mechanis, Ho Chi Minh City, July 2012.

[9] Rusydi, M.I., Sasaki, M. and Ito, S. (2014) Affine Transform to Reform Pixel Coordinate of EOG Signals for Controlling Robot Manipulators Using Gaze Motions. Sensor, 14, 10107-10123. http://dx.doi.org/10.3390/s140610107

[10] Okamaoto, T., Sasaki, M., Ito, S., Takeda, K. and Rusydi, M.I. (2013) Using Gaze Point and Blink Detection to Control Robot Arm by Use of the EOG Signal. Proceedings of the 22nd MAGDA Conference, Miyazaki, December 2013.

[11] Rusydi, M.I., Sasaki, M. and Ito, S. (2014) Calculate Target Position of Object in 3-Dimensional Area Based on the Perceived Location Using EOG Signals. Journal of Computer and Communications, 2. 